\title{
COLLINEAR CONTROL OF OSCILLATION MODES OF SPATIAL DOUBLE PENDULUM WITH VARIABLE GAIN
}

\author{
Alexey Smirnov \\ Institute for Problems in Mechanical Engineering \\ of Russian Academy of Sciences, \\ Peter the Great St. Petersburg Polytechnic University, \\ Russia \\ smirnov.alexey.1994@gmail.com
}

\author{
Boris Smolnikov \\ Institute for Problems in Mechanical Engineering \\ of Russian Academy of Sciences, \\ Peter the Great St. Petersburg Polytechnic University, \\ Russia \\ smolnikovba@yandex.ru
}

Article history:

Received 20.08.2021, Accepted 27.09.2021

\begin{abstract}
This article is devoted to the study of controlled movements of a spatial double pendulum with non-parallel cylindrical joints axes. The collinear control is used to swinging of the system by feedback. The most important property of collinear control is the ability of increasing system oscillations only on one oscillation mode. A modification of the collinear control law with variable gain depending on the energy level is investigated. It allows to control the system motions more flexible than in the case of constant gain. As a result, it is possible to observe a smooth transition from a linear oscillation mode to a nonlinear one with a gradual output to a steady oscillation motion with a given energy level. The obtained results are clearly illustrated by graph dependencies that demonstrate the swinging of the system on one oscillation mode from small to finite amplitudes.
\end{abstract}

\section{Key words}

pendulum, control, oscillations, frequency, mode, resonance.

\section{Introduction}

A large number of publications are devoted to the control of pendulum structures [Ananyevskiy, Fradkov, and Nijmeijer, 2008; Manevitch, Smirnov, and Romeo, 2016; Alyshev, Dudarenko, and Melnikov, 2018]. Herewith, control processes in pendulum systems with several degrees of freedom are of special interest. This is explained by the fact that it is necessary to pay here much more attention in the formation of control actions in order to achieve certain goals than in the study of systems with one degree of freedom. In particular, a number of works are related to control of the movements of a double pendulum and its modifications, and we should mention among them the following works [Lavrovskii, and Formalskii, 2001; Bogdanov, 2004; Awrejcewicz et al., 2008; Formalskii, 2014; Andreev, and Peregudova, 2015] published in the last two decades. We note that problem of the double pendulum has long and interesting history [Smirnov, and Smolnikov, 2020], and its research is of significant theoretical and practical interest. First of all, this is due to its application as an element of various multi-link structures in robotics, as well as in biodynamic problems. It should be emphasized that various spatial double pendulums attract the attention of specialists in the field of dynamics and motion control of pendulum systems [Bendersky, and Sandler, 2006; Xinjilefu, Hayward, and Michalska, 2009; Ludwicki, Awrejcewicz, and Kudra, 2015]. They have a much wider range of applicability than flat pendulums, and they are widely used in different practical purposes.

At the same time, it is very important to propose an approach to the formation of a fairly simple control law that allows such pendulum system to swing on each of its oscillation modes separately with their gradual transition to a nonlinear zone. This path should be considered rational, since during its implementation, all the energy supplied to the system will be directed to the development of only one oscillation mode, without exciting other modes. In this case, it is important also to ensure the gradual transition of the system to the required functioning mode which corresponds to a predetermined energy level and represents a regular periodic motion with sufficient large amplitude. These motions are the most valuable of all possible movement modes of nonlinear systems, because they are advisable for practical use in the operation of manipulators and other robotic devices. In this regard, the main purpose of this work is to construct and study a control with the indicated properties. 


\section{Spatial double pendulum}

We consider a double pendulum consisting of two pivotally connected identical mathematical pendulums of length $l$ with end loads of mass $m$. We assume that axes of the cylindrical joints of this pendulum form an acute angle $\alpha$ between themselves, so that they are not parallel to each other, and the double pendulum becomes spatial (Fig. 1). Kinetic and potential energies of this system were obtained in [Smirnov, and Smolnikov, 2021-4]:

$$
\begin{gathered}
T=\frac{1}{2} m l^{2}\left[\left(2+\cos ^{2} \alpha+\sin ^{2} \alpha \cos ^{2} \theta_{2}+\right.\right. \\
\left.\left.+2 \cos \theta_{2}\right) \dot{\theta}_{1}^{2}+2 \cos \alpha\left(1+\cos \theta_{2}\right) \dot{\theta}_{1} \dot{\theta}_{2}+\dot{\theta}_{2}^{2}\right], \\
\Pi=m g l\left[3-\left(2+\cos \theta_{2}\right) \cos \theta_{1}+\right. \\
\left.+\cos \alpha \sin \theta_{2} \sin \theta_{1}\right],
\end{gathered}
$$

where $\theta_{1}$ and $\theta_{2}$ are articulated rotation angles, which we consider as generalized coordinates, and $g$ is the acceleration due to gravity. We can write the equations of motion of this system in the following matrix form:

$$
\mathbf{A}(\boldsymbol{\theta}) \ddot{\boldsymbol{\theta}}+\mathbf{B}(\boldsymbol{\theta}, \dot{\boldsymbol{\theta}})+\mathbf{C}(\boldsymbol{\theta})=0 .
$$

Here $\boldsymbol{\theta}=\left[\theta_{1}, \theta_{2}\right]^{\mathrm{T}}$ is the column of generalized coordinates, and the matrix $\mathbf{A}(\boldsymbol{\theta})$, as well as columns $\mathbf{B}(\boldsymbol{\theta}, \dot{\boldsymbol{\theta}})$ and $\mathbf{C}(\boldsymbol{\theta})$ have the following representations:

$$
\begin{aligned}
& \mathbf{A}(\boldsymbol{\theta})=m l^{2}\left[\begin{array}{cc}
2+\cos ^{2} \alpha+ & \\
+\sin ^{2} \alpha \cos ^{2} \theta_{2}+ & \cos \alpha\left(1+\cos \theta_{2}\right) \\
+2 \cos \theta_{2} & \\
\cos \alpha\left(1+\cos \theta_{2}\right) & 1
\end{array}\right], \\
& \mathbf{B}(\boldsymbol{\theta}, \dot{\boldsymbol{\theta}})=m l^{2} \sin \theta_{2}\left[\begin{array}{r}
-\left(2\left(1+\sin ^{2} \alpha \cos \theta_{2}\right) \dot{\theta}_{1}+\right. \\
\left.+\cos \alpha \dot{\theta}_{2}\right) \dot{\theta}_{2} \\
\left(1+\sin ^{2} \alpha \cos \theta_{2}\right) \dot{\theta}_{1}^{2}
\end{array}\right], \\
& \mathbf{C}(\boldsymbol{\theta})=m g l\left[\begin{array}{c}
\left(2+\cos \theta_{2}\right) \sin \theta_{1}+\cos \alpha \sin \theta_{2} \cos \theta_{1} \\
\sin \theta_{2} \cos \theta_{1}+\cos \alpha \cos \theta_{2} \sin \theta_{1}
\end{array}\right] .
\end{aligned}
$$

In the same article [Smirnov, and Smolnikov, 2021-4], a linear model of this double pendulum was considered, and the linear matrix equation is

$$
\mathbf{A}_{0} \ddot{\boldsymbol{\theta}}+\mathbf{C}_{0} \boldsymbol{\theta}=0
$$

where matrix of inertial coefficients $\mathbf{A}_{0}$ and matrix of quasi-elastic coefficients $\mathbf{C}_{0}$ are constant symmetric matrices, and they have the form:

$$
\begin{gathered}
\mathbf{A}_{0}=m l^{2}\left[\begin{array}{cc}
5 & 2 \cos \alpha \\
2 \cos \alpha & 1
\end{array}\right] \\
\mathbf{C}_{0}=m g l\left[\begin{array}{cc}
3 & \cos \alpha \\
\cos \alpha & 1
\end{array}\right]
\end{gathered}
$$

As a result, the dimensionless frequencies $p_{s 0}=k_{s 0} / k$, where $k=\sqrt{g / l}$, and also the small oscillation modes
$\boldsymbol{\Theta}_{(s)}=\left[\Theta_{1 s}, \Theta_{2 s}\right]^{\mathrm{T}}$, which are characterized by the ratio $\beta_{s 0}$ between the oscillation amplitudes $\Theta_{2 s}$ and $\Theta_{1 s}$ of the rotation angles $\theta_{2}$ and $\theta_{1}$ in the pendulum joints, were determined, where $s=1,2$ :

$$
\begin{gathered}
p_{s 0}=\sqrt{\frac{2\left(1+\sin ^{2} \alpha\right) \pm \sqrt{2-\sin ^{2} \alpha}}{1+4 \sin ^{2} \alpha}}, \\
\beta_{s 0}=-\frac{3 \pm 2 \sqrt{2-\sin ^{2} \alpha}}{\cos 2 \alpha \pm \sqrt{2-\sin ^{2} \alpha}} \cos \alpha .
\end{gathered}
$$

It is necessary to define specific values $\Theta_{1 s}$ and $\Theta_{2 s}$ in the small oscillation mode for further actions, for example, $\Theta_{1 s}=1, \Theta_{2 s}=\beta_{s 0}$. We also need to introduce the normalizing coefficients of oscillation modes $N_{s}$, where

$$
\boldsymbol{\Theta}_{(s)}^{\mathrm{T}} \mathbf{A}_{0} \boldsymbol{\Theta}_{(s)}=N_{s}, \quad \boldsymbol{\Theta}_{(s)}^{\mathrm{T}} \mathbf{C}_{0} \boldsymbol{\Theta}_{(s)}=N_{s} k_{s 0}^{2},
$$

and the values $N_{s}$ can be calculated taking into account expressions (5) and (6).

\section{Formation of the motion control law}

It is known that the oscillation frequencies begin to drift with increase in amplitudes in a nonlinear system, and also the oscillation modes are gradually changing. In particular, the drift of frequencies and modes of spatial double pendulum was established in [Smirnov, and Smolnikov, 2021-3]. Therefore, the traditional methods of swinging of the system, that is, the organization of resonant oscillations, associated with harmonic excitation in the form of a given program in time with prescribed frequency from the outside and widely used in linear systems, are unsuitable here. This reason means that it is necessary to create control actions, according to the state when the frequency is fed to the excitation drive as a feedback signal. Such a controlled resonant mode is well known for systems with one degree of freedom, and its research begins from fundamental work [Andronov,

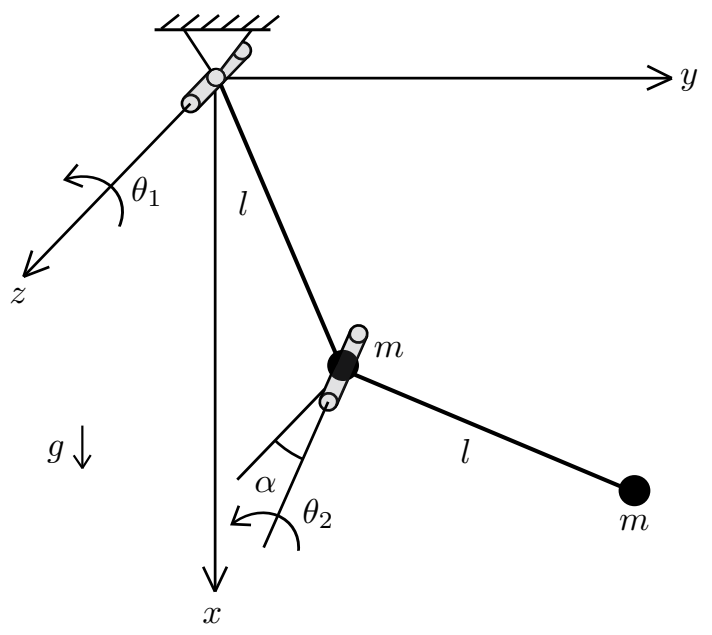

Figure 1. Spatial double pendulum 
Vitt, and Khaikin, 1966], where the concept of "autoresonance" was introduced for it. This controlled mode ensures that the frequency and phase of the feedback signal will exactly correspond to the current frequency and phase of the system oscillations, due to which all the energy entering the system will be transferred to it. However, this term remained practically unnoticed for many years, and the interest in this concept gradually increased only in the last several decades. For example, the works [Fradkov, 1999-1; Fradkov, 1999-2] are devoted to the feedback resonance. The main difficulty in the practical implementation of such resonance is the need to use a feedback loop in order to form the required drive control law. The formation of a control law using feedback can be carried out in many different ways for systems with one degree of freedom, when there will be a monotonic increase in the total energy $E=T+\Pi$ of the system.

The organization of swinging using feedback is more difficult in multidimensional nonlinear systems. We note that feedback resonance in such systems is studied in some works, for example, in [Efimov, Fradkov, and Iwasaki, 2013]. Serious difficulties arise here because these mechanical systems have several oscillatory degrees of freedom, i.e., several natural frequencies and modes. So that, it is necessary for swinging of the system in this case not only to increase the energy of the system, but also to be able to swing the system on one of its free oscillation modes separately. Otherwise, the energy supplied to the system will go to excite all modes at once, which will lead to a sharp decrease of the resonant properties of control and the quality of the motion processes. This means that the required control should not violate the linear oscillation modes of the system and allow a gradual transition of each oscillation mode to a corresponding nonlinear one, swinging the system from small amplitudes up to the sufficiently large ones. It is known that the specified property is possessed by the so-called "collinear control". From a mathematical point of view, collinear control involves the formation of a column of control actions $\mathbf{Q}$ collinear to the column of generalized impulses $\mathbf{K}=\partial T / \partial \dot{\boldsymbol{\theta}}$, i.e., in the form $\mathbf{Q}=\gamma \mathbf{K}$, where $\gamma$ is the gain factor. This control uses dynamic properties of the controlled system, imitating the inertial forces that arise in the process of its movement. Initially, it was proposed to control the motion of rigid bodies and various manipulators operating in the absence of external force fields [Merkin, and Smolnikov, 2003; Smolnikov, 1991]. Subsequently, this idea was applied to control the motion of pendulum systems that oscillate in the gravity field [Smirnov, and Smolnikov, 2017]. Herewith, the relation $\dot{E}=2 \gamma T$ is fulfilled, and it demonstrates the increasing character of the total energy. As a result, using the example of a flat double pendulum, it was shown that it is possible to swing this pendulum on each of the oscillation modes with a smooth transition from a linear to a nonlinear zone with the help of collinear control with small constant gain. In addition, it is possible to achieve an output to the conservative oscillatory motion with a periodicity by switching off the control when the de- sired energy level $E_{\infty}$ is reached. All of the techniques mentioned above enable to use the collinear control in a wide variety of areas of modern technology. It should also be emphasized that described controlled movements are apparently widespread in the animal world, where all running, swimming and flying animals perform their locomotion in a controlled resonant motion according to one of oscillation modes. Naturally, we also need to use these principles for locomotion of modern running androids [Leontev, Smirnov, and Smolnikov, 2019], that once again ensures the relevance of the proposed study.

\section{Collinear control with variable gain}

The collinear control with a constant gain $\gamma$ described above leads to a fairly rapid energy increasing in time, which stops abruptly when the value of $E_{\infty}$ is reached. So that, the question arises: how is it necessary to set the gain in order to achieve a smooth transition of the system to the steady motion with the desired energy level $E_{\infty}$ ? It is clear that it is necessary to gradually lower the gain as the energy approaches this value, thereby reducing the rate of its growth, that is, to set this gain in the form of a function depending on the state variables: $\gamma=\gamma(\boldsymbol{\theta}, \dot{\boldsymbol{\theta}})$. It is easy to understand that this dependence can be formed in the various ways. The most preferable option seems to be similar to that described in [Fradkov, 2007]:

$$
\gamma(\boldsymbol{\theta}, \dot{\boldsymbol{\theta}})=\gamma_{0} \frac{E_{\infty}-E(\boldsymbol{\theta}, \dot{\boldsymbol{\theta}})}{m g l},
$$

where the factor $m g l$ is introduced for dimensional reasons, so that constant value $\gamma_{0}$ has the same dimension as $\gamma$. It's seen that $\gamma$ gradually decreases to zero when the energy approaches the value $E_{\infty}$, therefore, the transition to the steady oscillation motion will be smooth in this situation. Considering that function (8) changes rather slowly, it can be assumed that this change has practically no effect on the main property of collinear control - preservation of the oscillation modes of the original conservative model. We recall that his circumstance is decisive, since the main task of the control action means the development of one or another oscillation mode of the system.

Let us turn to the study of controlled motions of the spatial double pendulum. We assume that control moments are formed using sensors installed in its joints, so we can write down the nonlinear matrix equation of the controlled motion of the system, adding to the right side (2) the column of control actions $\mathbf{Q}$ :

$$
\mathbf{A}(\boldsymbol{\theta}) \ddot{\boldsymbol{\theta}}+\mathbf{B}(\boldsymbol{\theta}, \dot{\boldsymbol{\theta}})+\mathbf{C}(\boldsymbol{\theta})=\mathbf{Q},
$$

wherein this column is formed according to the principle of collinear control, taking into account (8):

$$
\mathbf{Q}=\gamma(\boldsymbol{\theta}, \dot{\boldsymbol{\theta}}) \frac{\partial T}{\partial \dot{\boldsymbol{\theta}}}=\gamma_{0} \frac{E_{\infty}-E(\boldsymbol{\theta}, \dot{\boldsymbol{\theta}})}{m g l} \mathbf{A}(\boldsymbol{\theta}) \dot{\boldsymbol{\theta}},
$$




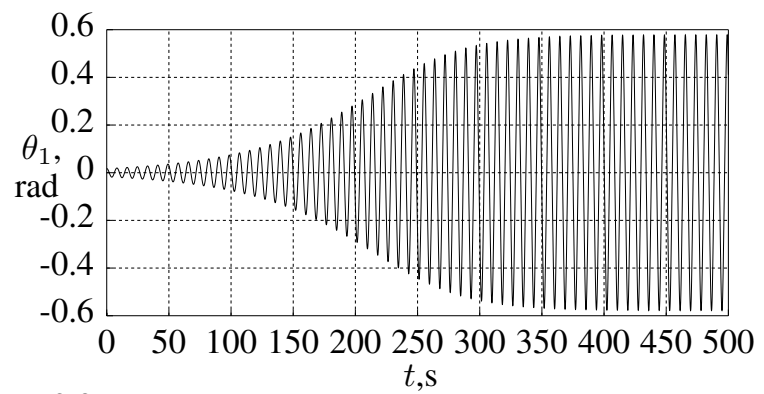

where the total energy is $E=T+\Pi$, and it is determined according to (1). The equation (9) can be solved using numerical methods. In order to excite a resonant motion on one of the oscillation modes of the system, it is necessary to set the initial conditions that correspond to the required mode in the linear model. For example, we can assume, for the simplicity, that at $t=0$ initial conditions have the form: $\boldsymbol{\theta}_{0}=a_{0} \boldsymbol{\Theta}_{(s)}, \dot{\boldsymbol{\theta}}_{0}=0$, where $a_{0}$ is the sufficiently small value. We restrict ourselves to the study of controlled motion only on the first oscillation mode, which is of the main practical significance.

\section{Discussion of the results of numerical research}

We turn to discussion of the results of numerical study at various values of angle $\alpha$ between the joint axes. First of all, we consider the special case of a flat double pendulum, when $\alpha=0$. The nonlinear modes for this situation were constructed in the work [Smirnov, and Smolnikov, 2021-2], where, in addition to the rotation angles $\theta_{1}$ and $\theta_{2}$ in joints, absolute deviation angles of pendulum links from the vertical $\varphi_{1}=\theta_{1}$ and $\varphi_{2}=\theta_{1}+\theta_{2}$ are used, which also are often considered as generalized coordinates. Fig. 2 shows the graph dependencies of angles $\theta_{1}, \theta_{2}$ and $\varphi_{2}$, as well as angular velocities $\dot{\theta}_{1}, \dot{\theta}_{2}$ and $\dot{\varphi}_{2}$ in time in the process of controlled movement. It can be seen that at the initial stage of motion all the presented quantities are rather small and gradually increase their amplitudes without changing their character. This means that oscillation mode is still linear at this stage. However, over time, there is a pronounced drift of the oscillation mode with its smooth transition to a nonlinear mode and the reaching a steady motion for all mentioned quantities. It can be seen that this movement retains a regular character and is a single-frequency one, while it is already very different from the motion on the linear oscillation mode, which has a harmonic, i.e., sinusoidal character. We note that the form of the nonlinear oscillation mode is in complete agreement with the results obtained in [Smirnov, and Smolnikov, 2021-2] using analytical methods based on asymptotic analysis, as well as in the case of numerical methods.

Now consider the controlled motion of a spatial double pendulum, for example, at $\alpha=\pi / 6$. The graph dependencies of angles $\theta_{1}$ and $\theta_{2}$, as well as angular velocities $\dot{\theta}_{1}$ and $\dot{\theta}_{2}$ in time are shown for this case in Fig. 3. It is easy to see that there is also a slow transition of the linear oscillation mode to the nonlinear one with the preservation of the single-frequency motion upon a detailed examination of presented graphs.

Finally, let us consider the case $\alpha=\pi / 2$ which corresponds to the so-called orthogonal double pendulum, when joint axes of both pendulums are orthogonal to each other. Then the matrices (5) are diagonal, and therefore, the oscillations in both degrees of freedom in this case are independent in linear conservative model. This means that one generalized coordinate can change oscil-

Figure 2. Control of a flat double pendulum ( $\alpha=0)$ 

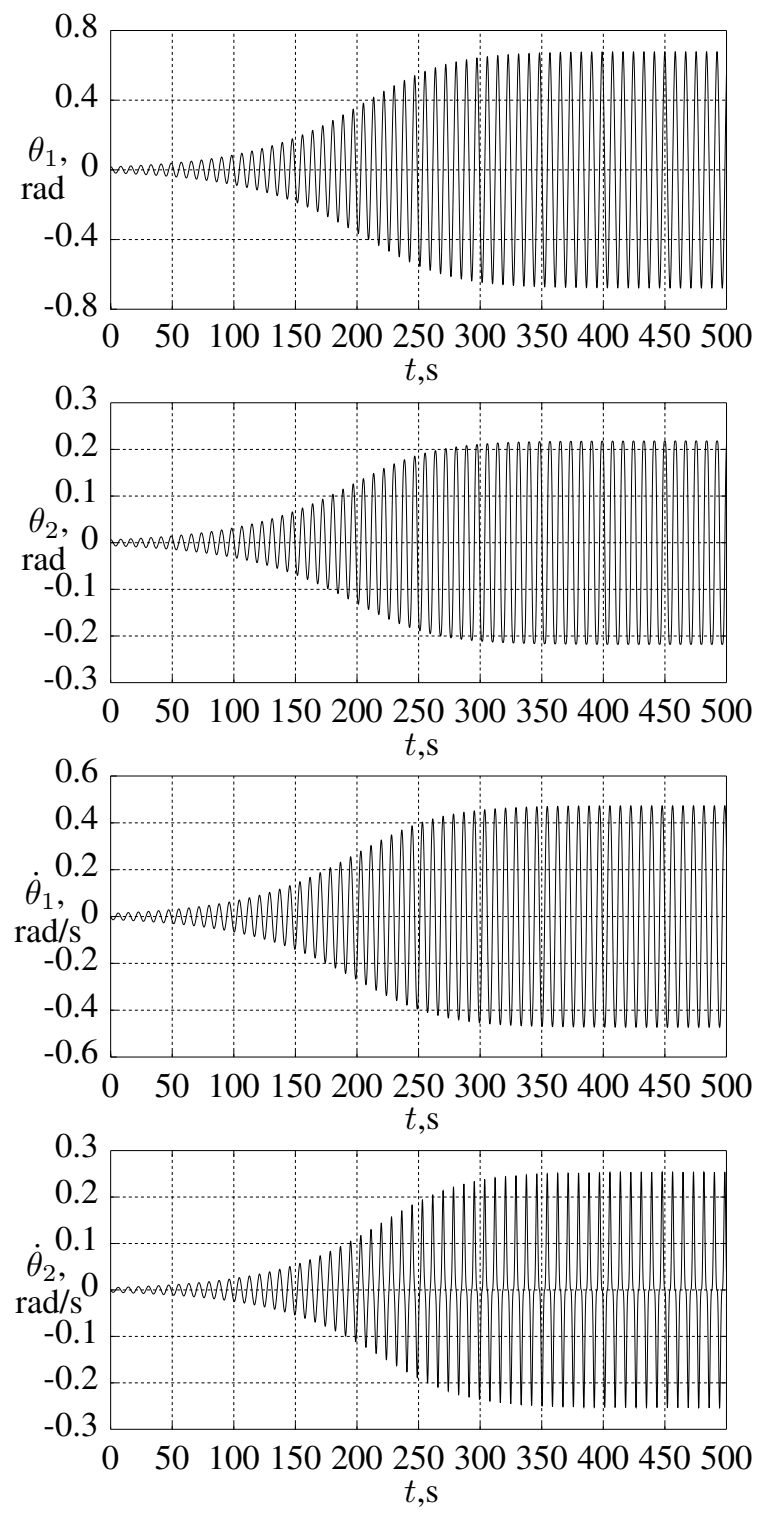

Figure 3. Control of a spatial double pendulum at $\alpha=\pi / 6$
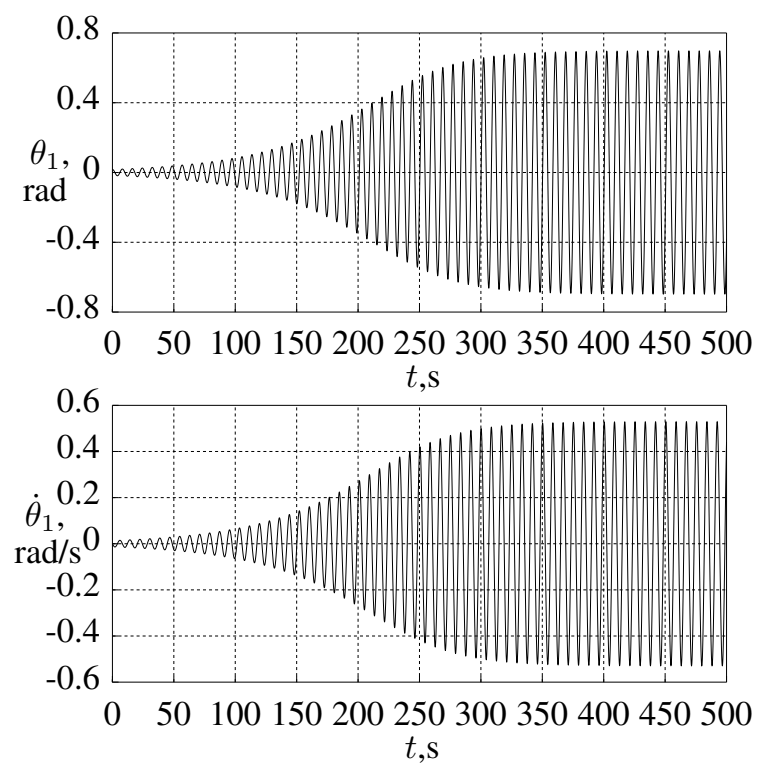

Figure 4. Control of an orthogonal double pendulum $(\alpha=\pi / 2)$ latory, while the second coordinate is equal to zero. It can be shown that it is possible to increase the amplitude of oscillations of the coordinate $\theta_{1}$ at zero value of the coordinate $\theta_{2}$, and the character of the controlled process will be completely analogous to the swinging of an ordinary mathematical pendulum studied in [Smirnov, and Smolnikov, 2021-1]. The graph dependencies of angle $\theta_{1}$ and angular velocity $\dot{\theta}_{1}$ in time are presented in Fig. 4, while the angle $\theta_{2}$ and angular velocity $\dot{\theta}_{2}$ are zero.

Thus, the following important conclusion can be made: the proposed collinear control law with variable gain really allows to swing the system on one of its oscillation modes with gradual transformation of this mode from linear to nonlinear one and the transition to a steady oscillatory motion corresponding to a given energy level.

\section{Analytical solution for small deviations}

We study the case of sufficiently small deviations, when the angles $\theta_{1}$ and $\theta_{2}$ can be considered as not so significant. It is possible to construct a simple analytical solution in this case, and it is an important feature. For this purpose, we can write down the expression of total energy using the quadratic approximation:

$$
E(\boldsymbol{\theta}, \dot{\boldsymbol{\theta}})=\frac{1}{2} \dot{\boldsymbol{\theta}}^{\mathrm{T}} \mathbf{A}_{0} \dot{\boldsymbol{\theta}}+\frac{1}{2} \boldsymbol{\theta}^{\mathrm{T}} \mathbf{C}_{0} \boldsymbol{\theta} .
$$

We will assume that it is required to bring the system to the harmonic oscillations on one of the modes $\boldsymbol{\Theta}_{(s)}$ with corresponding to it natural frequency $k_{s 0}$, i.e.,

$$
\boldsymbol{\theta}=\boldsymbol{\Theta}_{(s)} A \cos \psi, \quad \psi=k_{s 0} t+\vartheta_{s 0},
$$

where $A$ is a constant parameter that has the meaning of the amplitude and is also sufficiently the small one. We calculate the desired energy level corresponding to (12):

$$
E_{\infty}=\frac{1}{2} N_{s} k_{s 0}^{2} A^{2}
$$

where conditions (7) are used. For small deviations, the equation (9) can be simplified by reducing it to the form

$$
\mathbf{A}_{0} \ddot{\boldsymbol{\theta}}+\mathbf{C}_{0} \boldsymbol{\theta}=\mathbf{Q}(\boldsymbol{\theta}, \dot{\boldsymbol{\theta}}) .
$$

and here the column of control actions is

$$
\mathbf{Q}=\frac{\gamma_{0}}{2 m g l}\left(N_{s} k_{s 0}^{2} A^{2}-\dot{\boldsymbol{\theta}}^{\mathrm{T}} \mathbf{A}_{0} \dot{\boldsymbol{\theta}}-\boldsymbol{\theta}^{\mathrm{T}} \mathbf{C}_{0} \boldsymbol{\theta}\right) \mathbf{A}_{0} \dot{\boldsymbol{\theta}}
$$

We have excluded here the own nonlinearity of the system, which affects the oscillation frequency and mode in the first level of approximation, that we are not considering now, since we are interested in only the character of the oscillation growth here. Therefore, we have left only 

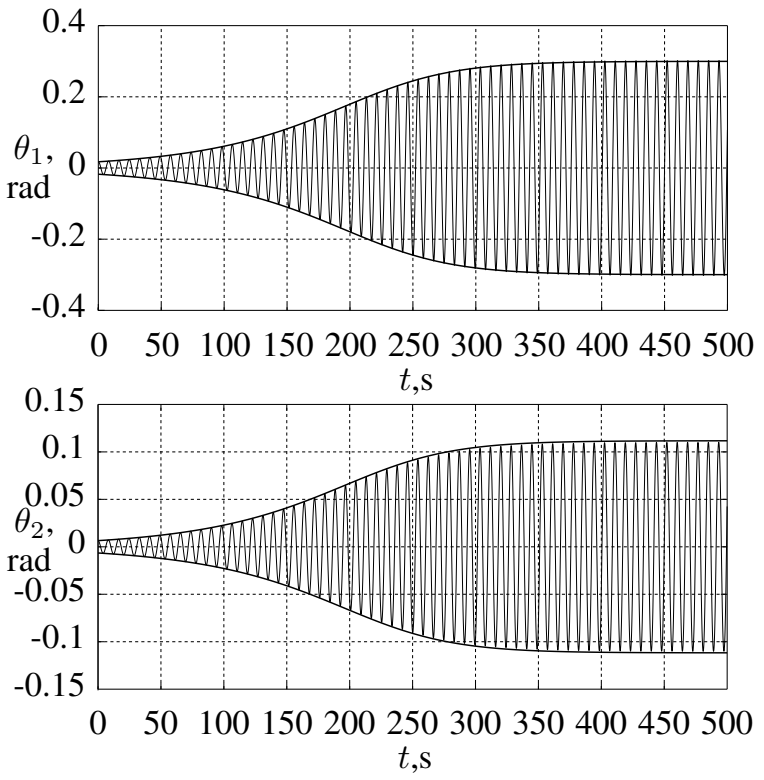

Figure 5. Control of a spatial double pendulum at $\alpha=\pi / 6$ (the case of small deviations)

nonlinear terms of the third order of smallness, arising due to control actions and directly affecting the increase in the amplitudes of oscillations. We will seek solution of equation (14) in the form:

$$
\boldsymbol{\theta}=\boldsymbol{\Theta}_{(s)} a \cos \psi, \quad \psi=k_{s 0} t+\vartheta_{s 0},
$$

where $a=a(t)$ is some unknown time function, which is to be determined. It should be found from the harmonic balance equation for oscillation mode $\boldsymbol{\Theta}_{(s)}$ [Bogoliubov, and Mitropolsky, 1961]:

$$
\int_{0}^{2 \pi} \boldsymbol{\Theta}_{(s)}^{\mathrm{T}}\left(\mathbf{A}_{0} \ddot{\boldsymbol{\theta}}+\mathbf{C}_{0} \boldsymbol{\theta}-\mathbf{Q}\right) \sin \psi d \psi=0 .
$$

We assume that $\dot{a}=F(a)$, where the function $F(a)$, as it is easy to understand, has the third order of smallness at values $a$ and $A=$ const, which we consider as values of the same order of smallness. Then, we calculate $\dot{\boldsymbol{\theta}}$ and $\ddot{\theta}$ according to (16) with the required precision:

$$
\begin{gathered}
\dot{\boldsymbol{\theta}}=\boldsymbol{\Theta}_{(s)}\left[F(a) \cos \psi-a \sin \psi k_{s 0}\right], \\
\ddot{\boldsymbol{\theta}}=\boldsymbol{\Theta}_{(s)}\left[-2 F(a) \sin \psi k_{s 0}-a \cos \psi k_{s 0}^{2}\right] .
\end{gathered}
$$

Let us calculate the column (15) up to the third order of smallness:

$$
\mathbf{Q}=-\frac{\gamma_{0} N_{s} k_{s 0}^{2}}{2 m g l}\left(A^{2}-a^{2}\right) a \sin \psi k_{s 0} \mathbf{A}_{0} \boldsymbol{\Theta}_{(s)} .
$$

Substituting now formulas (16), (18) and (19) into the equation (17), we obtain after some transformations the following expression:

$$
F(a)=\frac{\gamma_{0} N_{s} k_{s 0}^{2}}{4 m g l} a\left(A^{2}-a^{2}\right) .
$$

Thus, we obtain the equation to determine $a(t)$ :

$$
\dot{a}=\kappa_{s} a\left(A^{2}-a^{2}\right), \quad \kappa_{s}=\frac{\gamma_{0} H_{s} p_{s 0}^{2}}{4},
$$

where it is taken into account that $p_{s 0}=k_{s 0} / k$, and also dimensionless normalization coefficients are introduced: $H_{s}=N_{s} /\left(m l^{2}\right)$. Taking the initial conditions in the previous form $\boldsymbol{\theta}_{0}=a_{0} \boldsymbol{\Theta}_{(s)}, \dot{\boldsymbol{\theta}}_{0}=0$ and referring to the formulas (16) and (18), and, in addition, taking into account that $F(a)$ is small in comparison with $a$, we can approximately obtain that $\vartheta_{s 0}=0$, and $a=a_{0}$ at $t=0$. Solving (21), we finally find the desired dependence:

$$
a(t)=\frac{a_{0} A e^{\kappa_{s} A^{2} t}}{\sqrt{A^{2}+a_{0}^{2}\left(e^{2 \kappa_{s} A^{2} t}-1\right)}} .
$$

It is seen from (22) that for $t \rightarrow \infty$ we have $a \rightarrow A$, i.e., $E \rightarrow E_{\infty}$, as expected. As a result, for small deviations, the controlled motion of a spatial double pendulum is approximately described by the formulas (16) and (22). Fig. 5 shows the graphs of controlled motion at $\alpha=\pi / 6$ at sufficiently small angles $\theta_{1}$ and $\theta_{2}$. The envelope lines, which characterize the oscillation amplitudes of specified angles, are plotted according to the obtained formulas. It is seen that these lines are in complete agreement with the results of numerical integration.

Thus, the obtained analytical results enable to assess the character of increase in the oscillation amplitudes within the framework of the considered simplified model. We emphasize once again that despite the approximations carried out and the absence of a drift of the frequency and oscillation mode in this model, it still remains nonlinear due to the control actions.

\section{Conclusion}

Summarizing the results of the study of controlled motion of a spatial double pendulum, we can conclude that collinear control with a variable gain, as well as collinear control with constant gain, allows to transfer all the energy supplied to the system to the growth of oscillations only on one oscillation mode. This means, that it is possible to excite resonant oscillations in a given oscillation mode with the help of such control, and besides the oscillation amplitudes of both articulated rotation angles increase and tend to the predetermined level over time. As a result, it is possible to observe the system movement on a nonlinear oscillation mode characterized by a single frequency. Of course, the proposed method can be applied to any other mechanical system, especially in the field of robotics and biodynamics.

Finally, it is necessary to note the invaluable contribution of I. I. Blekhman to many of the issues discussed in this article. His remarkable papers are devoted to the theory of nonlinear oscillations, vibrational mechanics, resonance phenomenon, cybernetical physics and many other fields of applied mechanics and control processes [Blekhman, 2000; Blekhman, and Fradkov (eds), 2001; Blekhman, 2012; Blekhman, and Sorokin, 2018]. 


\section{References}

Alyshev, A., Dudarenko, N., and Melnikov V. (2018) Parametric identification of reaction wheel pendulums with adaptive control. Cybernetics and Physics, 7(2), pp. 57-65.

Ananyevskiy, M. S., Fradkov, A. L., and Nijmeijer, H. (2008) Control of two pendulums with energy constraints. In 6th Euromech nonlinear oscillations conference (ENOC-2008), Saint Petersburg, Russia, June, 30-July, 42008.

Andreev, A. S., and Peregudova, O. A. (2015) On control for double-link manipulator with elastic joints. Russian Journal of Nonlinear Dynamics, 11(2), pp. 267-277.

Andronov, A., Vitt, A., and Khaikin, S. (1966) Theory of Oscillators. Pergamon Press, Oxford, 848 p.

Awrejcewicz, J., Reshmin, S. A., Wasilewski, G., and Kudra, G. (2008) Swing up a double pendulum by simple feedback control. In 6th Euromech nonlinear oscillations conference (ENOC-2008), Saint Petersburg, Russia, June, 30-July, 42008.

Bendersky, S., and Sandler, B. (2006) Investigation of a spatial double pendulum: an engineering approach. Discrete Dynamics in Nature and Society, pp. 1-22.

Blekhman, I. I. (2000) Vibrational mechanics. World Scientific, Singapore, $510 \mathrm{p}$.

Blekhman, I. I. (2012) Oscillatory strobodynamics a new area in nonlinear oscillations theory, nonlinear dynamics and cybernetical physics. Cybernetics and Physics, 1(1), pp. 5-10.

Blekhman, I. I., and Fradkov, A. L. (eds) (2001) Control of Mechatronic Vibrational Units. Nauka, Saint Petersburg, $278 \mathrm{p}$.

Blekhman, I. I., and Sorokin, V. S. (2018) On a "deterministic" explanation of the stochastic resonance phenomenon. Nonlinear Dynamics, 93(2), pp. 767-778.

Bogdanov, A. (2004) Optimal Control of a Double Inverted Pendulum on a Cart. Technical Report CSE-04006.

Bogoliubov, N. N., and Mitropolsky, Y. A. (1961) Asymptotic Methods in the Theory of Non-Linear Oscillations. Gordon and Breach, New York, $537 \mathrm{p}$.

Efimov, D., Fradkov A., and Iwasaki, T. (2013) Exciting multi-DOF systems by feedback resonance. Automatica, 49(6), pp. 1782-1789.

Formalskii, A. M. (2014) Motion control of unstable objects. Fizmatlit, Moscow, $232 \mathrm{p}$.

Fradkov, A. L. (1999) Exploring nonlinearity by feedback. Physica D., 128(2-4), pp. 159-168.

Fradkov, A. L. (1999) Investigation of physical systems by feedback. Automation and Remote Control, 60(3), pp. 471-483.

Fradkov, A. L. (2007) Cybernetical physics. From Control of Chaos to Quantum Control. Springer-Verlag, Berlin, Heidelberg, 242 p.

Lavrovskii, E. K., and Formalskii, A. M. (2001) The optimal control synthesis of the swinging and damping of a double pendulum. Journal of Applied Mathematics and Mechanics, 65 (2), pp. 219-227.
Leontev, V. A., Smirnov, A. S. and Smolnikov, B. A. (2019) Collinear control of dissipative double pendulum. Journal of Robotics and Technical Cybernetics, 7 (1), pp. 65-70.

Ludwicki, M., Awrejcewicz, J., and Kudra, G. (2015) Spatial double physical pendulum with axial excitation: computer simulation and experimental set-up. International Journal of Dynamics and Control, 3, pp. 1-8.

Manevitch, L. I., Smirnov, V. V., and Romeo, F. (2016) Non-Stationary Resonance Dynamics Of The Harmonically Forced Pendulum. Cybernetics and Physics, 5 (3), pp. 91-95.

Merkin, D. R., and Smolnikov, B. A. (2003) Applied problems of rigid body dynamics. SPbSU publishing, Saint Petersburg, 534 p.

Smirnov, A. S., and Smolnikov, B. A. (2017) Resonance oscillations control of the non-linear mechanical systems based on the principles of biodynamics. Mashinostroenie $i$ inzhenernoe obrazovanie, 4(53), pp. 11-19.

Smirnov, A. S., and Smolnikov, B. A. (2020) Double pendulum research history. History of Science and Engineering, 12, pp. 3-12.

Smirnov, A. S., and Smolnikov, B. A. (2021) Collinear control of single-link manipulator motion with variable gain. In Youth and Science: actual problems of fundamental and applied research, Materials of the IV All-Russian scientific conference of students, postgraduates and young scientists, Komsomolsk-onAmur, April 12-16, vol. 2, pp. 70-73.

Smirnov, A. S., and Smolnikov, B. A. (2021) Nonlinear oscillation modes of double pendulum. In IOP Conference Series: Materials Science and Engineering, International Conference of Young Scientists and Students "Topical Problems of Mechanical Engineering" (ToPME 2020) 2nd-4th December 2020, Moscow, Russia, vol. 1129, 012042.

Smirnov, A. S., and Smolnikov, B. A. (2021) Nonlinear oscillation modes of spatial double pendulum. In Journal of Physics: Conference Series, The International Scientific Conference on Mechanics "The Ninth Polyakhov's Reading" (ISCM) 9-12 March 2021, Saint Petersburg, Russian Federation, vol. 1959, 012046.

Smirnov, A. S., and Smolnikov, B. A. (2021) Oscillations of Double Mathematical Pendulum with Noncollinear Joints. In Lecture Notes in Mechanical Engineering, 9th conference on Modern Engineering: Science and Education (MMESE), 25 June 2020, Saint Petersburg, pp. 185-193.

Smolnikov, B. A. (1991) The problems of mechanics and robotoptimization. Nauka, Moscow, $232 \mathrm{p}$.

Xinjilefu, Hayward, V., and Michalska, H. (2009) Stabilization of the Spatial Double Inverted Pendulum Using Stochastic Programming Seen as a Model of Standing Postural Control. In 9th IEEE-RAS International Conference on Humanoid Robots, pp. 367-372. 